\title{
Vibrational Energy Relaxation of Isotopically Labeled Amide I Modes in Cytochrome $c$ : Theoretical Investigation of Vibrational Energy Relaxation Rates and Pathways
}

\author{
Hiroshi Fujisaki**,,$*$ and John E. Straub $\$, \S, \perp$ \\ Department of Chemistry, Boston University, 590 Commonwealth Avenue, Boston, Massachusetts 02215, \\ Institute of Physical and Theoretical Chemistry, J. W. Goethe University, Max-von-Laue Strasse 7, 60438 \\ Frankfurt am Main, Germany, and Department of Chemistry and Biochemistry, Montana State University, \\ Bozeman, Montana 59717
}

Received: April 4, 2007; In Final Form: July 8, 2007

\begin{abstract}
With use of a time-dependent perturbation theory, vibrational energy relaxation (VER) of isotopically labeled amide I modes in cytochrome $c$ solvated with water is investigated. Contributions to the VER are decomposed into two contributions from the protein and water. The VER pathways are visualized by using radial and angular excitation functions for resonant normal modes. Key differences of VER among different amide I modes are demonstrated, leading to a detailed picture of the spatial anisotropy of the VER. The results support the experimental observation that amide I modes in proteins relax with subpicosecond time scales, while the relaxation mechanism turns out to be sensitive to the environment of the amide I mode.
\end{abstract}

\section{Introduction}

Amide I vibrational modes in proteins or peptides are localized around the peptide backbone and can be a sensitive probe of protein structure and dynamics. Mainly localized around the $\mathrm{CO}$ bond of the backbone with large oscillator strength, the amide I modes detected with infrared (IR) spectroscopy have been studied for a variety of proteins and peptides. ${ }^{1-5}$ Recently 2D-IR spectroscopy ${ }^{6-8}$ has been utilized to decipher (anharmonic) coupling between vibrational modes including the amide I mode., ${ }^{9,10}$ The interpretation of the associated spectra is not necessarily simple, and will benefit from the development of increasingly accurate theoretical models. ${ }^{11-13}$

Combining MD simulations with ab initio calculations, several theoretical groups have recently devised sophisticated methods to characterize the effect of inhomogeneity on the dephasing time, $T_{2}$, of the amide I mode, using small peptides in water. ${ }^{14-18}$ Anharmonic frequency calculations have been performed for a peptide-like molecule, $N$-methylacetamide (NMA), in vacuum ${ }^{19-22}$ and in water. ${ }^{17}$ For the amide I mode of NMA in water, theoretical investigations of vibrational energy relaxation (VER) have been carried out using a quasiclassical method $^{23}$ and a quantum mechanical perturbation method. ${ }^{24}$ However, while there have been many experimental studies ${ }^{25-27}$ of VER of amide I modes in proteins, related to $T_{1}$, there are relatively few theoretical studies.

In this work, we study the VER properties of amide I modes in a protein, cytochrome $c$, in water, and clarify the VER pathways using quantum-mechanical time-dependent perturbation theory. ${ }^{24}$ This is a continuation of our previous work on VER of a CD stretching mode in cytochrome $c .^{28,29}$ The amide I modes studied were isotopically labeled according to IR experiment. ${ }^{30,31} \mathrm{We}$ decompose the VER rate into two compo-

\footnotetext{
* Address correspondence to this author. E-mail: fujisaki@ theochem.uni-frankfurt.de.

$\dagger$ Boston University.

$\doteqdot$ J. W. Goethe University.

$\S$ Montana State University.

${ }^{\perp}$ Electronic address: straub@bu.edu.
}

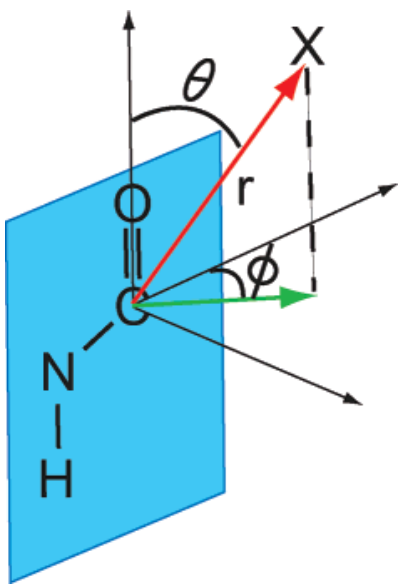

Figure 1. The polar coordinates $(r, \theta, \phi)$ for an atom $X$ used to calculate the radial and angular excitation functions of a normal mode. The origin of the coordinate system is the $C_{\beta}$ atom of the amide I mode. The sheet represents the amide plane.

nents: one from the protein and the other from water (solvent). We identify the resonant modes that contribute most significantly to the VER rate, and introduce distribution-like functions for those modes to visualize the spatial anisotropy of VER as pathways in 3-dimensional space.

This paper is organized as follows. In Section II, our timedependent perturbation method is briefly described, and several methods to visualize the VER pathways in protein and water are proposed. In Section III, we examine the results of VER rates by comparing with the Maradudin-Fein formula, SASA, localization length, and experimental results. Furthermore, we mention the anisotropy of VER from excited amide I modes into protein as well as into water. In Section IV, we summarize and discuss the future extension of our work.

\section{Methods}

A. VER Rate Formula. We briefly summarize the quantum mechanical perturbation theory employed in this work. We assume that normal mode coordinates $q_{\alpha}$ provide a good 
description of the system dynamics for the observables studied. We further assume that the potential energy function can be Taylor expanded up to the third and fourth order anharmonic terms with respect to normal mode coordinates including only the relaxing mode $q_{\mathrm{S}}{ }^{24}$

$$
\begin{gathered}
H=\mathbf{H}_{\mathbf{S}}+\mathbf{H}_{\mathrm{B}}-q_{\mathrm{S}} \delta \mathbf{F}+q_{\mathrm{S}}{ }^{2} \delta \mathbf{G} \\
\mathbf{H}_{S}=\frac{p_{\mathrm{S}}{ }^{2}}{2}+V\left(q_{\mathrm{S}}\right) \\
\mathbf{H}_{\mathrm{B}}=\sum_{\alpha} \frac{p_{\alpha}{ }^{2}}{2}+\frac{\omega_{\alpha}{ }^{2} q_{\alpha}{ }^{2}}{2} \\
\delta \mathbf{F}=\sum_{\alpha, \beta} C_{\mathrm{S} \alpha \beta}\left(q_{\alpha} q_{\beta}-\left\langle q_{\alpha} q_{\beta}\right\rangle\right) \\
\delta \mathbf{G}=\sum_{\alpha, \beta} C_{\mathrm{SS} \alpha \beta}\left(q_{\alpha} q_{\beta}-\left\langle q_{\alpha} q_{\beta}\right\rangle\right)+\sum_{\alpha} C_{\mathrm{SS} \alpha} q_{\alpha}
\end{gathered}
$$

where $\mathbf{H}_{\mathrm{S}}\left(\mathbf{H}_{\mathrm{B}}\right)$ is the system (bath) Hamiltonian, and $C_{\mathrm{S} \alpha \beta}$ $\left(C_{\mathrm{SS} \alpha \beta}\right)$ are the third (fourth) order coupling terms. (This is related to the three-mode representation of a quartic force field. ${ }^{22,32}$ ) From the von Neumann-Liouville equation, a reduced density matrix for the relaxing mode is derived by using the time-dependent perturbation theory after tracing over the bath degrees of freedom. (A similar result has been derived from the path integral formulation of quantum mechanics by Okazaki and co-workers. ${ }^{33}$ ) The Markov approximation is usually employed to derive a simplified Bloch-Redfield-type equation after introducing the density of states for the bath. However, to describe the initial stage of the quantum dynamics, we do not invoke the Markov approximation. Using this approach, we are able to avoid assumptions related to the lifetimes of the vibrational modes of the bath-the so-called "line width problem," 28 used in the Markov approximation.

When the relaxing mode is excited to the $v=1$ state, the VER is described by the decay of the reduced density matrix element $\left(\rho_{\mathrm{S}}\right)_{11}(t)$. We define the temporal VER rate as $R(t)=$ $\mathrm{d}\left(\rho_{\mathrm{S}}\right)_{11}(t) / \mathrm{d} t$, which is approximately written as ${ }^{24}$

$$
\begin{gathered}
R(t) \simeq \frac{2}{\hbar^{2}} \sum_{\alpha, \beta}\left[C_{--}^{\alpha \beta} \dot{u}_{t}\left(\tilde{\omega}_{\mathrm{S}}-\omega_{\alpha}-\omega_{\beta}\right)+\right. \\
C_{++}^{\alpha \beta} \dot{u}_{t}\left(\tilde{\omega}_{\mathrm{S}}+\omega_{\alpha}+\omega_{\beta}\right)+ \\
\left.C_{+-}^{\alpha \beta} \dot{u}_{t}\left(\tilde{\omega}_{\mathrm{S}}-\omega_{\alpha}+\omega_{\beta}\right)\right] \equiv \sum_{\alpha, \beta} R^{\alpha \beta}(t)
\end{gathered}
$$

where $\dot{u}_{t}(\Omega)=\sin \Omega t / \Omega, \tilde{\omega}_{\mathrm{S}}$ is the anharmonicity-corrected system frequency, $\omega_{\alpha}$ is the bath mode (harmonic) frequency, and the coefficients $C_{++}^{\alpha \beta}, C_{+-}^{\alpha \beta}, C_{--}^{\alpha \beta}$ are derived from the nonlinear coupling constants $C_{\mathrm{S} \alpha \beta}$ and $C_{\mathrm{SS} \alpha \beta} \cdot{ }^{24} R^{\alpha \beta}(t)$ is each component of the VER rate corresponding to modes $\alpha$ and $\beta$. We note that the first term in eq 6 dominates in the formula because of the resonance condition, i.e., $\dot{u}_{t}(\Omega)$ becomes large when $\tilde{\omega}_{S}-\omega_{\alpha}-\omega_{\beta} \simeq 0$.

Because of non-Markovian properties, $R(t)$ is not necessarily a constant. If the Markov assumption holds, $R(t)$ becomes a rate constant. In the case of the amide I modes in cytochrome $c$, we have confirmed that a "VER rate" can be defined as a time average of $R(t)$ after a certain transient time $t_{\mathrm{tr}}$

$$
\bar{R} \equiv \frac{1}{T-t_{\mathrm{tr}}} \int_{t_{\mathrm{tr}}}^{T} R(t) \mathrm{d} t=\sum_{\alpha, \beta} \bar{R}^{\alpha \beta}
$$

with $T \simeq 1.0 \mathrm{ps}$ and $t_{\mathrm{tr}} \simeq 0.5 \mathrm{ps}$. With this procedure, the averaged VER time is obtained as $T_{1} \simeq 1 / \bar{R}$. The final rate formula is similar to the Maradudin-Fein (MF) formula, ${ }^{28,34}$ and both formulas lead to a rate. An essential difference is that we avoid invoking the Markov approximation and introducing the phenomenological line width parameter. However, the MF formula has been widely employed in the literature, ${ }^{28,34}$ and it would be interesting to examine the usefulness of the MF formula based on our formula. Hence we introduce the MF rate as

$$
R^{\mathrm{MF}}=\frac{2}{\hbar^{2}} \sum_{\alpha, \beta} C_{--}^{\alpha \beta} \frac{\delta}{\delta^{2}+\left(\tilde{\omega}_{\mathrm{S}}-\omega_{\alpha}-\omega_{\beta}\right)^{2}}
$$

where $\delta$ is the line width parameter, which will be discussed below.

B. Analysis of VER Pathways. As shown in eq 7, the VER rate $\bar{R}$ can be decomposed into components $\bar{R}^{\alpha \beta}$. This result allows us to examine which modes (combination) contribute significantly to the VER rate. To develop a detailed picture of VER in a complex molecular system, we utilize the normal mode eigenvectors $U_{k}^{\alpha}$ which satisfy

$$
\sum_{k l} U_{k}^{\alpha} F_{k l} U_{l}^{\beta}=\omega_{\alpha}^{2} \delta_{\alpha \beta}
$$

where $\alpha(k)$ represents the normal mode index (Cartesian coordinate index) and $F_{k l}$ is the mass-weighted Hessian matrix.

We first introduce the localization length of the normal $\operatorname{mode}^{35,36}$

$$
L^{\alpha}=\left[\sum_{k}\left|U_{k}^{\alpha}\right|^{4}\right]^{-1}
$$

which measures how a normal mode extends in a system in terms of the number of atoms. This measure (also called inverse participation ratio) is used to quantify the localization of the amide I modes.

We are interested in evaluating contributions from protein and water to the value and mechanism of VER. We assign modes to water (protein) if the condition $\sum_{k \in \text { water }}\left|U_{k}^{\alpha}\right|^{2}>0.5$ $\left(\sum_{k \in \text { protein }}\left|U_{k}^{\alpha}\right|^{2}>0.5\right)$ is satisfied. Here $k \in$ water (protein) means that the summation is taken only for water (protein) degrees of freedom. Hence $\bar{R}^{\alpha \beta}$ can be decomposed into the (1) protein-protein contribution (if $\alpha$ and $\beta \in$ protein), (2) protein-water contribution (if $\alpha(\beta) \in$ protein and $\beta(\alpha) \in$ water), and (3) water-water contribution (if $\alpha$ and $\beta \in$ water).

We next introduce radial and angular excitation distribution functions for each normal mode $\alpha$ as

$$
\begin{gathered}
g^{\alpha}(r)=\sum_{k \in \Delta \Gamma}\left|U_{k}^{\alpha}\right|^{2} \\
h^{\alpha}(\theta, \phi)=\sum_{k \in \Delta \Omega}\left|U_{k}^{\alpha}\right|^{2}
\end{gathered}
$$

where $\Delta \Gamma$ and $\Delta \Omega$ are the bins for the radial direction (whose median is $r$ ) and the bins for the cubic angle (whose median is $\theta, \phi)$, respectively (see Figure 1). The summation is only taken over these bins. We refer to $g^{\alpha}(r)$ and $h^{\alpha}(\theta, \phi)$ as excitation distribution functions because they represent coarse-grained spatial information about the excitation of a normal mode. 

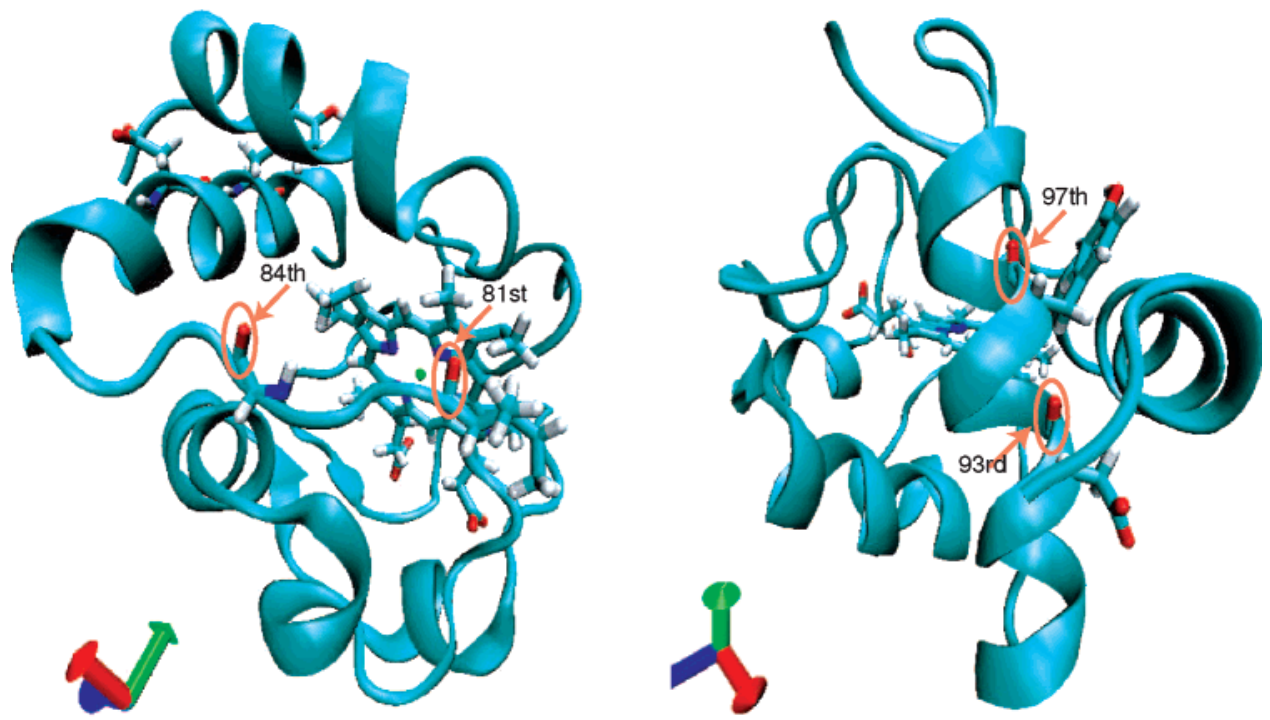

Figure 2. Left: 81st and 84th residues in cytochrome $c$ in a loop region. Right: 93rd and 97th residue in cytochrome $c$ in an $\alpha$ helical region. The cartoon represents the protein, and the licorice the four residues. (The water molecules are excluded for simplicity.) VMD (visual molecular dynamics) was used to generate these figures..$^{52}$

Note that these are different from the ordinary distribution functions (the absolute value does not have physical meaning), and are not directly related to experimental observables (such as neutron scattering functions). These functions are specifically introduced for visualizing the VER pathways. Other approaches are possible. For example, Dijkstra and Knoester visualized normal modes of $\beta$-hairpin and $\beta$-sheet peptides using a color map in their analysis of IR and 2D-IR spectra. ${ }^{35}$

We define the average excitation distribution functions for resonant modes as

$$
\begin{gathered}
\bar{g}(r)=\frac{1}{N_{\text {res }}} \sum_{\alpha \in \text { res }} g^{\alpha}(r) \\
\bar{h}(\theta, \phi)=\frac{1}{N_{\text {res }}} \sum_{\alpha \in \text { res }} h^{\alpha}(\theta, \phi)
\end{gathered}
$$

where $\alpha \in$ res indicates that the summation is taken only for resonant modes, meaning those modes with $\bar{R}^{\alpha \beta}>R_{\mathrm{th}} . N_{\text {res }}$ is the number of such resonant modes. We took $R_{t \mathrm{~h}} \simeq 0.04 \mathrm{ps}^{-1}$ for purposes of illustration. As indicated in eq 6, when a system mode $\mathrm{S}$ is excited, the excess energy mainly flows to resonant (bath) modes $\alpha$ and $\beta$ via Fermi resonance $\left(\tilde{\omega}_{\mathrm{S}}-\omega_{\alpha}-\omega_{\beta} \simeq\right.$ $0)$. We can explore the spatial content of such resonant modes using the excitation distribution functions.

Sagnella and Straub introduced another way to visualize the anisotropy of energy flow in a protein, ${ }^{36}$ and the differences are (1) we consider quantum mechanical energy flow whereas their calculation is classical and (2) we employ the resonant normal modes whereas they used excess kinetic energies as the definition of the energy flow pathway. Okamoto and Nagaoka utilized an approach derived from hydrodynamics to visualize the anisotropic energy flow from a diatomic molecule in water, ${ }^{37}$ though their work is also based on classical mechanics. Much similar to the present work is that by Mikami and Okazaki, who devised a strategy for analyzing anisotropy of energy flow using quantum mechanics, ${ }^{38}$ but the method is tailored for VER problems of a diatomic molecule. In this work, we take the naive approach described above to visualize resonant normal modes.

C. Numerical Procedure. We used CHARMM ${ }^{39}$ to construct and simulate the system consisting of cytochrome $c$ and solvent water. CHARMM facilities were utilized to calculate dynamics, normal modes, solvent accessible surface area (SASA), etc., and the computational details have been presented elsewhere. ${ }^{40}$ After equilibration, a $100 \mathrm{ps}$ trajectory was generated and configurations were saved every 1 ps. From the 100 sample configurations, we calculated the VER rate using eq 7 and the results were averaged.

Application of our formula starts from the decomposition of the system Hamiltonian using normal modes as in eq 1. However, computation of normal modes for the full system is prohibitive. Hence we take a reduced system strategy: We deleted all atoms except those in a spherical region $r<R_{\mathrm{c}}$ (see Figure 1) where $R_{\mathrm{c}}=12 \AA .{ }^{24}$ For the reduced system, we carried out instantaneous normal mode analysis ${ }^{23,41}$ to obtain the normal mode frequencies $\omega_{\alpha}$ (imaginary frequencies are neglected). We computed the anharmonic coefficients $C_{\mathrm{S} \alpha \beta}, C_{\mathrm{SS} \alpha \beta}$ using a finite difference approximation ${ }^{28}$ along the normal mode directions. The summation in eq 7 was taken only for the bath modes with $\omega_{\alpha}>\omega_{\mathrm{c}}$, where we used $\omega_{\mathrm{c}}=100 \mathrm{~cm}^{-1}$ as the frequency cutoff.

TABLE 1: Protein and Water Contributions to the VER Rate for the Amide I Modes of Four Residues in Cytochrome $c$ in Water $^{a}$

\begin{tabular}{ccccccrr}
\hline residue & $\bar{R}$ & $\bar{R}_{\mathrm{pp}}$ & $\bar{R}_{\mathrm{pw}}$ & $\bar{R}_{\mathrm{ww}}$ & $R^{\mathrm{MF}}$ & IPR & SASA(CO) \\
\hline 81st & $2.29(0.03)$ & $0.95(0.02)$ & $1.21(0.02)$ & $0.13(0.00)$ & $2.24(0.03)$ & $9.54(1.03)$ & $\sim 80$ \\
84th & $2.36(0.03)$ & $0.99(0.02)$ & $1.31(0.03)$ & $0.06(0.00)$ & $2.29(0.04)$ & $8.26(0.95)$ & $\sim 100$ \\
93rd & $2.27(0.03)$ & $1.18(0.02)$ & $1.05(0.02)$ & $0.04(0.00)$ & $2.24(0.03)$ & $3.92(0.03)$ & $\sim 90$ \\
97th & $1.75(0.03)$ & $1.46(0.03)$ & $0.30(0.01)$ & $0.00(0.00)$ & $1.82(0.03)$ & $4.71(0.13)$ & $\sim 70$
\end{tabular}

${ }^{a}$ The total rate is $\bar{R}$, and the protein-protein, protein-water, water-water contribution are denoted as $\bar{R}_{\mathrm{pp}}, \bar{R}_{\mathrm{pw}}$, and $\bar{R}_{\mathrm{ww}}$, respectively. The value derived from the Maradudin-Fein formula is denoted as $R^{\mathrm{MF}}$. The units are $\mathrm{ps}^{-1}$ for the VER rate. The inverse participation ratio (localization length) of the amide I modes and solvent accessible surface area (SASA) for the CO atoms are also shown. The units for SASA are $\AA^{2}$. The number in parentheses represents the standard error of the mean. 
(a)

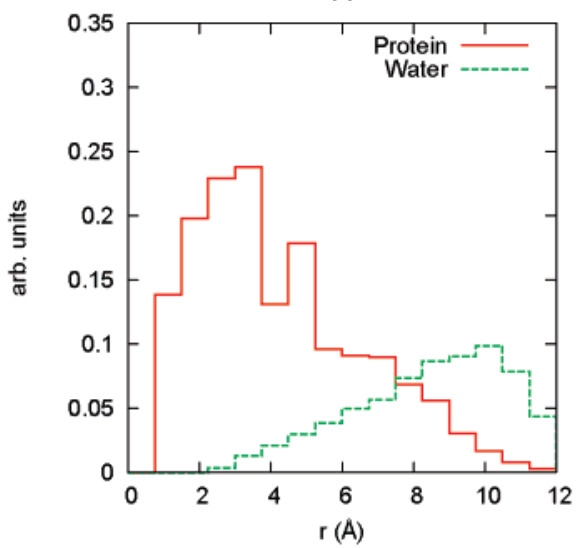

(c)

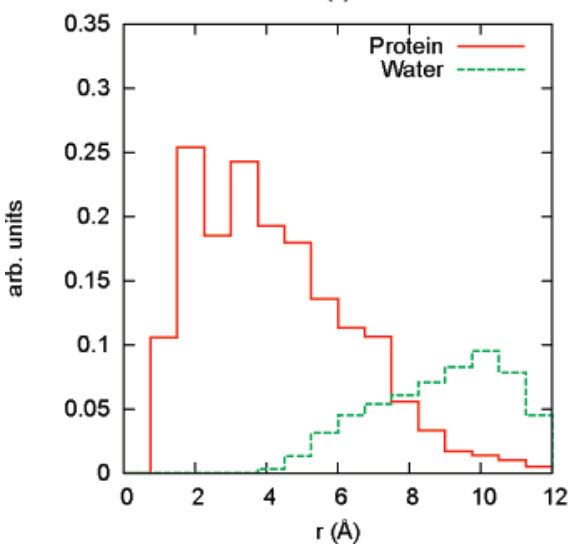

(b)

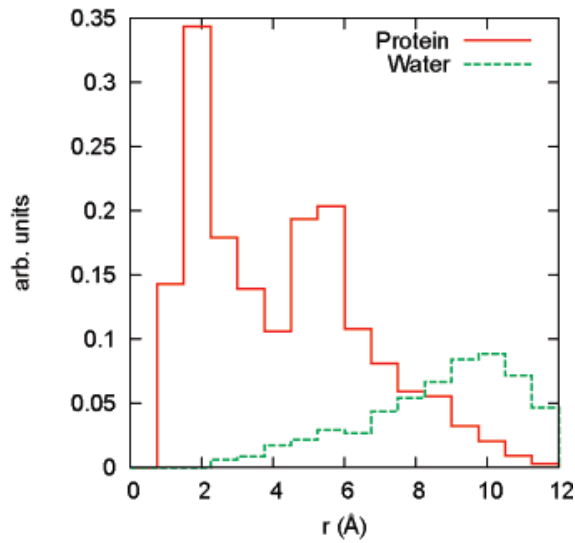

(d)

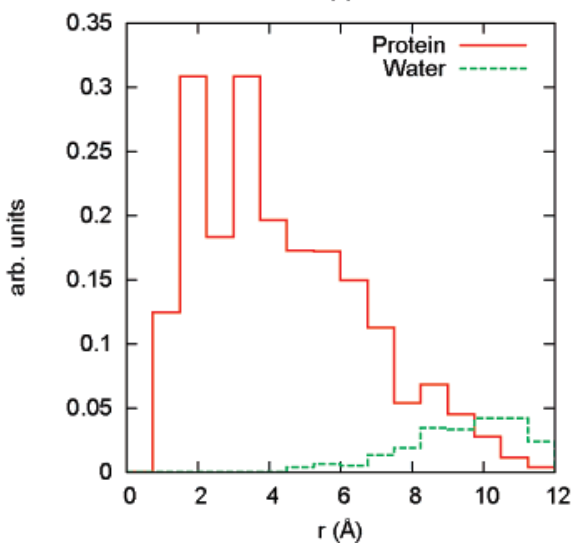

Figure 3. Radial excitation functions for the resonant normal modes of protein and water for the (a) 81st, (b) 84th, (c) 93rd, and (d) 97th residues, represented in arbitrary units.

This is a reasonable approximation, as we have observed that the low-frequency modes do not contribute significantly to the VER rate.

In the CHARMM program, we isotopically labeled one $\mathrm{CO}$ bond in a residue as ${ }^{13} \mathrm{C}={ }^{18} \mathrm{O}^{30,31}$ and we examined four different cases as described below. It was expected that isotopical labeling should localize the amide I mode, but this is not necessarily the case as shown below.

\section{Results and Discussions}

A. Protein and Water Contribution to the VER Rate. We isotopically labeled four residues: 81st, 84th, 93rd, and 97th (see Figure 2). The first two belong to a loop region of cytochrome $c$ whereas the latter two belong to a $\alpha$ helical region. By examing SASA (Table 1), we see that the CO atoms of the 84th residue are most exposed to solvent whereas those of the 97 th residue are most buried in the protein.

In Table 1, we observe that the VER rates are $\simeq 2 \mathrm{ps}^{-1}\left(T_{1} \simeq\right.$ $0.5 \mathrm{ps}$ ) for all the cases. This is similar to the VER rate of the amide I mode of $N$-methylacetamide in heavy water $(\simeq 2.0$ $\left.\mathrm{ps}^{-1}\right)^{24-26}$ though the 97 th residue has a slightly slower rate. It is interesting to note that the MF formula, eq 8, gives rather "accurate" values with an appropriate line width parameter $\delta$ $=3 \mathrm{~cm}^{-1}$. This result seems to validate the use of the MF formula and to indicate that the MF formula is sufficient to describe VER. However, note that an appropriate value of the line width parameter $\delta$ cannot be determined in advance.

Looking into the contributions from protein and water, we observe that the protein-water contribution is significantly less for the 97th residue compared to other residues (81st, 84th, and 93rd). This is because the water surrounds the $\mathrm{CO}$ bond less in this case (see Figure 2) as quantified by SASA for the CO bond (see Table 1). There seems to be a correlation between SASA and $\bar{R}_{\text {pw. }}$. However, as shown below, the water motion $8 \AA$ away from the $\mathrm{CO}$ bond can be involved in the VER processes. Hence the interpretation is not so simple.

We also investigate this point in terms of the localization length, eq 10, for the amide I modes. The amide I mode is expected to be localized on a $\mathrm{CO}$ bond only but this is necessarily the case as shown in Table 1. For the four residues we examined here, the amide I mode extends over from 4 to 10 atoms. For more delocalized modes, the protein-water or water-water contributions are expected to be large because there are more contacts with water. However, this expectation is not validated in a strict sense as shown in Table 1 . We know that the Fermi resonance parameter ${ }^{22,29}$ can strictly describe the VER pathways in molecules, but for the present system it is difficult to interpret the result based on some chemical intuition.

Note that the protein-protein contributions for the 81st, 84th, and 93rd residues are smaller than that for the 97th residue. However, due to the protein-water contribution, the VER rates of the former three amide I modes are faster than that of the latter. We conclude that VER of the former amide I modes is protein-mediated, whereas that of the latter residue is watermediated, which should have some experimental consequences as mentioned below.

How do our calculated VER rates obtained in this theoretical study compare with experimentally derived values? Hochstrasser and co-workers obtained subpicosecond VER time scales for amide I modes in several proteins. ${ }^{25,26}$ Zanni and co-workers also observed subpicosecond VER time scales for isotopically labeled amide I modes in a membrane protein. ${ }^{30}$ The experi- 
(a)

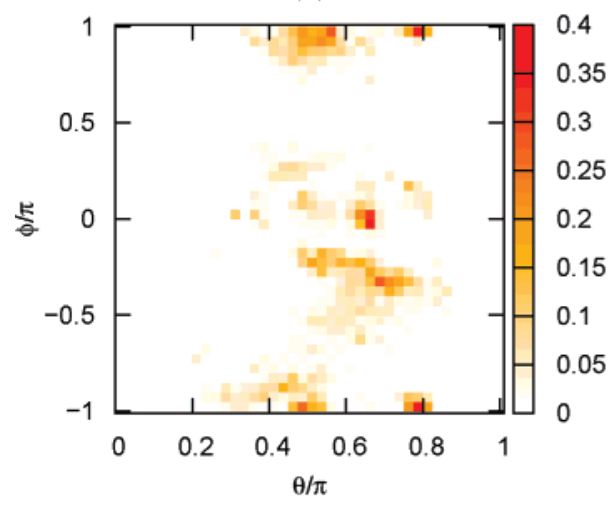

(c)

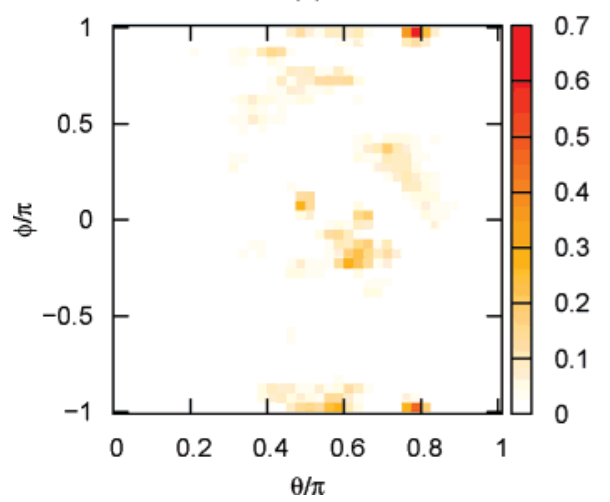

(b)

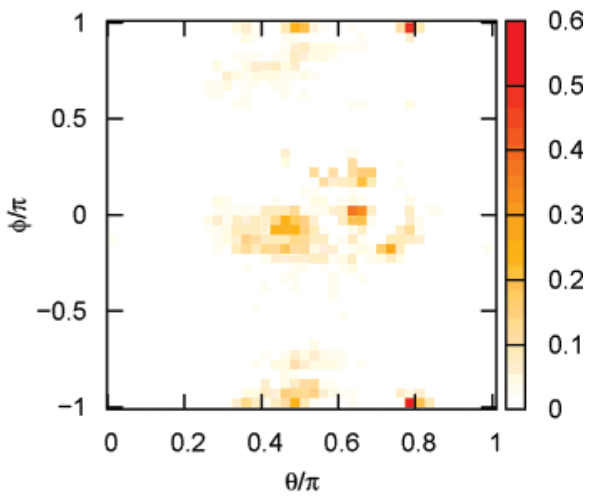

(d)

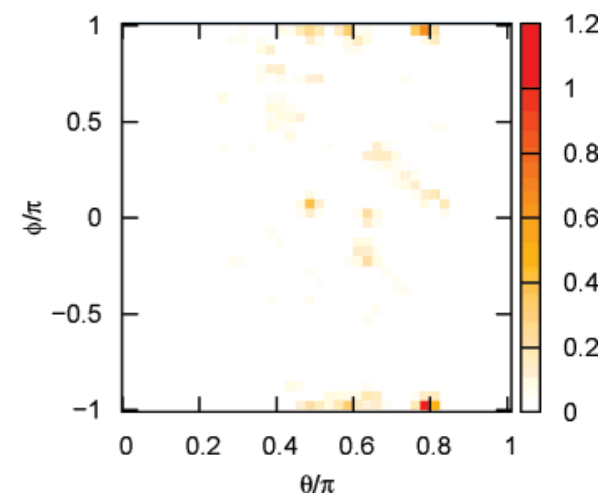

Figure 4. Angular excitation functions for the resonant normal modes of the protein for the (a) 81st, (b) 84th, (c) 93rd, and (d) 97th residues, represented in arbitrary units.

mentally derived VER times are comparable to our theoretical results (subpicosecond). However, note that although the total VER rates are similar, the physical mechanism is sensitive to the specific structural environment of the amide I mode as discussed above (Table 1). By using multidimensional spectroscopy, ${ }^{7,8,25,26,30}$ it should be possible to discriminate the protein and water contributions to VER separately in experiment.

We have recently studied the solvent effects on heme cooling in myoglobin. ${ }^{42} \mathrm{We}$ found that the mechanism for VER is dependent on resonance as well as structural anisotropy that modulates mode coupling. It will be interesting to investigate the solvent effects on VER of amide I modes by changing the nature of the solvent within the quantum mechanical perturbation theory (while the previous work ${ }^{42}$ is based on classical mechanics).

B. Visualization of VER Pathways. We have used the previously defined excitation functions, eqs 13 and 14, to scrutinize the spatial anisotropy of the VER pathways. The radial excitation function in Figure 3 shows each (protein or water) contribution to VER pathways along the radial direction. The peaks around $r \simeq 2 \AA$ correspond to the nearest neighbor intramolecular pathways involving the motion of the $\mathrm{NH}$ bond (see Figure 1). Since the density of the protein has a peak around $r \simeq 6 \AA$, the radial excitation function also has a peak or shoulder around the same location.

Looking at the water contributions, we found that the excitation functions have significant values at large distances (>8 $\AA$ ), indicating that VER is mediated by distant water molecules. On the other hand, Mikami and Okazaki found that only the water molecules of the first solvation shell contribute to VER in the case of a solvated diatomic molecule. ${ }^{38}$ We note that there are significant differences between the relaxation of an amide I mode in a protein and that of a solvated diatomic molecule. For VER (or energy transfer) to occur, the overlap of resonant normal modes is needed. ${ }^{43}$ In the case of a diatomic molecule, the stretching mode is, by definition, localized around the bond, and mainly couples to the neighboring water molecules. However, in the case of amide I modes in solvated proteins, the amide I modes are delocalized as shown in Table 1. Many resonant modes couple to such amide I modes, which can contain delocalized (collective) water motions. Further theoretical and experimental studies are needed to clarify the role of such delocalized modes on VER in proteins.

Finally we examine the angular excitation functions in Figures 4 and 5 to understand the VER pathways along the angular directions. We observe that the VER of the amide I modes is spatially anisotropic. For the protein (Figure 4), the resonant modes are mainly localized around $\phi=0$ or $\pm \pi$, which indicates that VER occurs in the peptide backbone plane (see Figure 1). For the 81 st and 84 th residues, belonging to a loop structure, the value around $\phi \approx 0$ enhances because the amide plane is planer and the intramolecular contribution comes from such a direction. For the 93rd and 97th residues, belonging to an $\alpha$ helical structure, the value around $\phi \approx 0$ diminishes because the amide I plane is bent.

On the other hand, for the water (Figure 5), the angular excitation function is rather broad, and these distributions are similar to the conventional distribution function of water around the $\mathrm{CO}$ bond (not shown here). However, the excitation functions are less uniform compared to the conventional distribution function of water, implying the anisotropy of VER into water. While these excitation functions cannot be directly measured, it is of significant interest to examine the anisotropy of energy flow in proteins by using experimental methods such as multidimensional spectroscopy. 7,8,25,26,30 
(a)

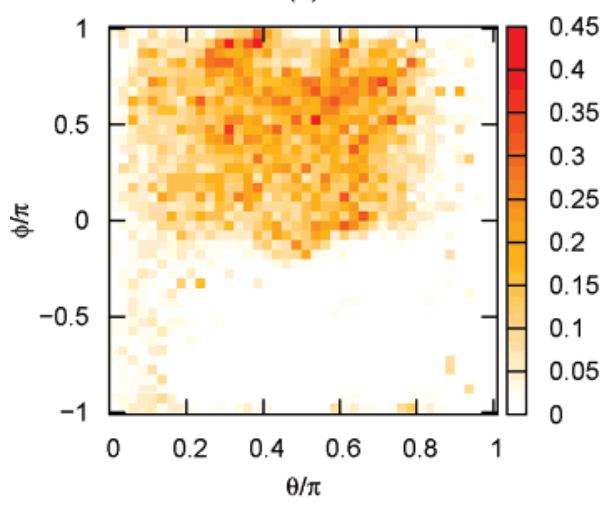

(c)

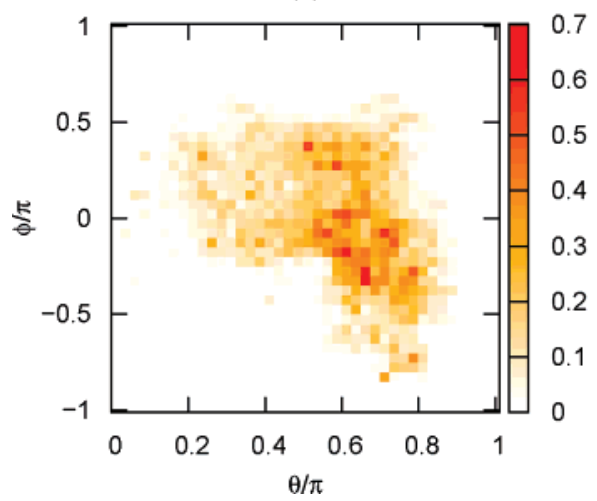

(b)

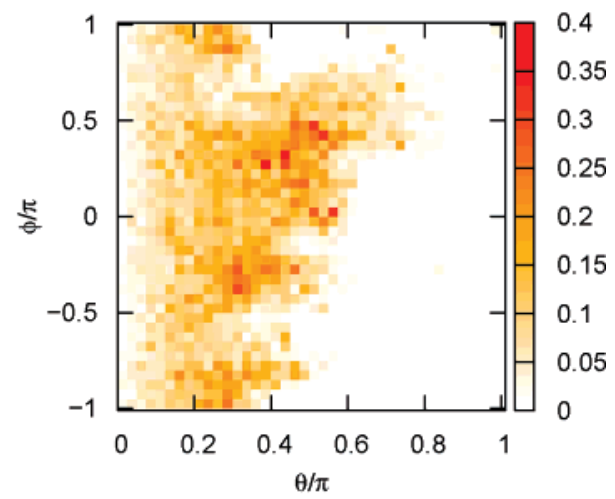

(d)

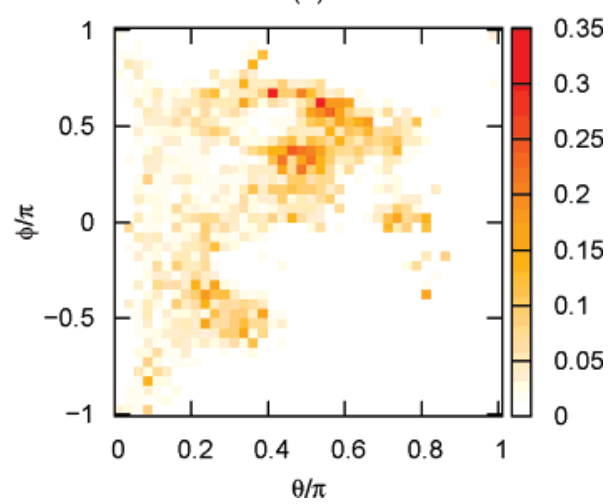

Figure 5. Angular excitation functions for the resonant normal modes of water for the (a) 81st, (b) 84th, (c) 93rd, and (d) 97th residues, represented in arbitrary units.

Recently Dlott and co-workers ${ }^{44}$ and Hamm and co-workers ${ }^{45}$ devised a new experimental technique to clarify energy transfer pathways in molecular systems. This method also may be combined with our analysis to clarify the water contribution of VER in a protein.

\section{Concluding Remarks}

Using the time-dependent perturbation formula we developed in our previous paper, we have investigated the vibrational energy relaxation (VER) of amide I modes in cytochrome $c$ solvated with water. We observed that the VER rates are subpicosecond for the four residues we examined here, which are in accord with previous experiment for other proteins. We have decomposed VER into separate contributions from protein and water, and further projected the resonant modes onto radial and angular excitation functions. Although the total VER rate is similar, the detailed mechanism of VER is different; there are protein-mediated pathways and water-mediated pathways depending on which residue is excited. There is anisotropy of VER for the water-mediated pathways as well as for the proteinmediated pathways, implying experimental consequences by multidimensional spectroscopy or other means.

Anharmonicity is a key issue in VER calculations. We note that our simulated dynamics are based on the CHARMM force field, in which vibrational anharmonicity has been underestimated. There is some evidence that the anharmonic coupling calculated using the force field can be comparable to that derived from $a b$ initio calculations (Fujisaki, H.; Yagi, K.; Hirao, K.; Straub, J. E., unpublished). However, without such a direct comparison, it is unclear how accurate the results of our force field calculations are. Nevertheless, the use of empirical energy functions is currently the only feasible way to characterize VER in large molecules. To expand on these studies, we must develop a new methodology to investigate the quantum dynamics of the amide I mode with ab initio potential surfaces. As first steps, we investigated $N$-methylacetamide in vacuum using the VCI method $^{22}$ and in water cluster using the perturbation method (Zhang, Y.; Fujisaki, H.; Straub, J. E., unpublished) on ab initio potentials. How to extend these studies to larger systems using multiresolution methods ${ }^{46,47}$ or QM/MM methods ${ }^{48-50}$ will be our focus in the near future.

Another important and related topic that should be pursued by such ab initio methods is the effect of polarization. ${ }^{14-18}$ Morita and Kato clarified that polarization is important for VER of a stretching mode in azide ion in water, ${ }^{51}$ and this also might be the case for VER of amide I modes in a protein. In particular, it is important to investigate some portion of a protein where the internal electric field is large and the polarization effect is expected to be enhanced.

Acknowledgment. We thank Y. Zhang for a useful suggestion on the relationship between the VER rate and SASA and anonymous referees for useful suggestions. We are also grateful to Prof. G. Stock, Prof. D. M. Leitner, Prof. K. Hirao, Prof. B. Brutschy, Prof. J. Wachtveitl, Dr. K. Yagi, and Dr. A. Furuhama for useful discussions. We thank the National Science Foundation (CHE-0316551), Boston University's Center for Computer Science for generous support to our research, and the Department of Chemistry and Biochemistry at Montana State University for generous support and hospitality.

\section{References and Notes}

(1) Krimm, S.; Bandekar, J. Adv. Protein Chem. 1986, 38, 181.

(2) (a) Torii, H.; Tasumi, M. J. Chem. Phys. 1992, 96, 3379. (b) Torii, H.; Tasumi, M. J. Chem. Phys. 1992, 97, 92.

(3) Barth, A.; Zscherp, C. Q. Rev. Biophys. 2002, 35, 369. 
(4) Silva, R. A. G. D.; Barber-Armstrong, W.; Decatur, S. M. J. Am. Chem. Soc. 2003, 125, 13674.

(5) Gnanakaran, S.; Hochstrasser, R. M.; Garcia, A. E. Proc. Natl. Acad. Sci. U.S.A. 2004, 101, 9229.

(6) (a) Tanimura, Y.; Mukamel, S. J. Chem. Phys. 1993, 99, 9496. (b) Mukamel, S. Principles of Nonlinear Optical Spectroscopy; Oxford University Press: New York, 1995.

(7) Zanni, M. T.; Hochstrasser, R. M. Curr. Opin. Struct. Biol. 2001 11,516 .

(8) Woutersen, S.; Hamm, P. J. Phys. Condens. Matter 2002, 14, 1035.

(9) Wang, J. P.; Hochstrasser, R. M. J. Phys. Chem. B 2006, 110 3798.

(10) DeFlores, L. P.; Ganim, Z.; Ackley, S. F.; Chung, H. S.; Tokmakoff, A. J. Phys. Chem. B 2006, 110, 18973.

(11) Ishizaki, A.; Tanimura, Y. J. Chem. Phys. 2006, 125, 084501.

(12) Nagata, Y.; Tanimura, Y.; Mukamel, S. J. Chem. Phys. 2007, 126, 204703.

(13) Torii, H. J. Phys. Chem. B 2007, 111, 5434.

(14) (a) Ham, S.; Hahn, S.; Lee, C.; Kim, T. K.; Kwak, K.; Cho, M. J. Phys. Chem. B 2004, 108, 9333. (b) Ham, S.; Hahn, S.; Lee, C.; Cho, M. J. Phys. Chem. B 2005, 109, 11789.

(15) (a) Schmidt, J. R.; Corcelli, S. A.; Skinner, J. L. J. Chem. Phys. 2004, 121, 8887. (b) Li, S.; Schmidt, J. R.; Corcelli, S. A.; Lawrence, C. P.; Skinner, J. L. J. Chem. Phys. 2006, 124, 204110.

(16) Zhuang, W.; Abramavicius, D.; Hayashi, T.; Mukamel, S. J. Phys. Chem. B 2006, 110, 3362.

(17) Hayashi, T.; la Cour Jansen, T.; Zhuang, W.; Mukamel, S. J. Phys. Chem. A 2005, 109, 64

(18) Gorbunov, R. D.; Nguyen, P. H.; Kobus, M.; Stock, G. J. Chem. Phys. 2007, 126, 054509.

(19) Gregurick, S. K.; Chaban, G. M.; Gerber, R. B. J. Phys. Chem. A 2002, 106, 8696.

(20) Bounouar, M.; Scheurer, Ch. Chem. Phys. 2006, 323, 87.

(21) Kaledin, A. L.; Bowman, J. M. J. Phys. Chem. A 2007, 111, 5593.

(22) Fujisaki, H.; Yagi, K.; Hirao, K.; Straub, J. E. Chem. Phys. Lett. 2007, 443, 6 .

(23) Nguyen, P. H.; Stock, G. J. Chem. Phys. 2003, 119, 11350.

(24) Fujisaki, H.; Zhang, Y.; Straub, J. E. J. Chem. Phys. 2006, 124 144910.

(25) Hamm, P.; Lim, M. H.; Hochstrasser, R. M. J. Phys. Chem. B 1998 102,6123 .

(26) Zanni, M. T.; Asplund, M. C.; Hochstrasser, R. M. J. Chem. Phys. 2001, 114, 4579.

(27) (a) Xie, A.; van der Meer, L.; Hoff, W.; Austin, R. H. Phys. Rev. Lett. 2000, 84, 5435. (b) Austin, R. H.; Xie, A.; van der Meer, L.; Redlich, B.; Lindgard, P. A.; Frauenfelder, H. Phys. Rev. Lett. 2005, 94, 128101.

(28) (a) Fujisaki, H.; Bu, L.; Straub, J. E. Adv. Chem. Phys. 2005, 130B, 179. (b) Fujisaki, H.; Bu, L.; Straub, J. E. In Normal Mode Analysis: Theory and Applications to Biological and Chemical Systems; Cui, Q., Bahar, I., Eds.; Chapman and Hall/CRC Press: Boca Raton, FL, 2005. (c) Fujisaki, H.; Straub, J. E. Proc. Natl. Acad. Sci. U.S.A. 2005, 102, 6726.
(29) Cremeens, M.; Fujisaki, H.; Zhang, Y.; Zimmermann, J.; Sagle, L. B.; Matsuda, S.; Dawson, P. E.; Straub, J. E.; Romesberg, F. E. J. Am. Chem. Soc. 2006, 128, 6028.

(30) (a) Mukherjee, P.; Krummel, A. T.; Fulmer, E. C.; Kass, I.; Arkin, I. T.; Zanni, M. T. J. Chem. Phys. 2004, 120, 10215. (b) Mukherjee, P.; Kass, I.; Arkin, I. T.; Zanni, M. T. J. Phys. Chem. B 2006, 110, 24740.

(31) Arkin, I. T. Curr. Opin. Chem. Biol. 2006, 10, 394.

(32) Yagi, K.; Hirao, K.; Taketsugu, T.; Schmidt, M. W.; Gordon, M. S. J. Chem. Phys. 2004, 121, 1383.

(33) (a) Shiga, M.; Okazaki, S. J. Chem. Phys. 1998, 109, 3542. (b) Shiga, M.; Okazaki, S. J. Chem. Phys. 1999, 111, 5390. (c) Mikami, T.; Shiga, M.; Okazaki, S. J. Chem. Phys. 2001, 115, 9797. (d) Mikami, T.; Okazaki, S. J. Chem. Phys. 2004, 121, 10052.

(34) (a) Yu, X.; Leitner, D. M. J. Phys. Chem. B 2003, 107, 1698. (b) Leitner, D. M. Adv. Chem. Phys. 2005, 130B, 205. (c) Leitner, D. M.; Havenith, M.; Gruebele, M. Int. Rev. Phys. Chem. 2006, 25, 553.

(35) Dijkstra, A. G.; Knoester, J. J. Phys. Chem. B 2005, 109, 9787.

(36) Sagnella, D. E.; Straub, J. E. J. Phys. Chem. B 2001, 105, 7057.

(37) Okamoto, T.; Nagaoka, M. Chem. Phys. Lett. 2005, 407, 444.

(38) Mikami, T.; Okazaki, S. J. Chem. Phys. 2003, 119, 4790.

(39) (a) Brooks, B. R.; Bruccoleri, R. E.; Olafson, B. D.; States, D. J.; Swaminathan, S.; Karplus, M. J. Comput. Chem. 1983, 4, 187. (b) MacKerell, A. D., Jr.; Brooks, B.; Brooks, C. L., III; Nilsson, L.; Roux, B.; Won, Y.; Karplus, M. In The Encyclopedia of Computational Chemistry; Schleyer, P. v. R., et al., Eds.; John Wiley \& Sons: Chichester, UK, 1998; Vol. 1, p271.

(40) Bu, L.; Straub, J. E. Biophys. J. 2003, 85, 1429.

(41) (a) Stratt, R. M. Acc. Chem. Res. 1995, 28, 201. (b) Keyes, T. J. Phys. Chem. A 1997, 101, 2921.

(42) Zhang, Y.; Fujisaki, H.; Straub, J. E. J. Phys. Chem. B 2007, 111, 3243 .

(43) (a) Moritsugu, K.; Miyashita, O.; Kidera, A. Phys. Rev. Lett. 2000, 85, 3970. (b) Moritsugu, K.; Miyashita, O.; Kidera, A. J. Phys. Chem. B 2003, 107, 3309 .

(44) Wang, Z.; Pakoulev, A.; Dlott, D. D. Science 2002, 296, 2201.

(45) Botan, V.; Backus, E. H. G.; Pfister, R.; Moretto, A.; Toniolo, C.; Nguyen, P. H.; Stock, G.; Hamm, P. Proc. Natl. Acad. Sci. U.S.A. 2007, 104, 12749.

(46) Rauhut, G. J. Chem. Phys. 2004, 121, 9313.

(47) Yagi, K.; Hirata, S.; Hirao, K. Theor. Chem. Acc. 2007, 118, 681.

(48) Lin, S. Z.; Schmidt, J. R.; Corcelli, S.; Lawrence, C. P.; Skinner, J. L. J. Chem. Phys. 2006, 124, 204110.

(49) Hirata, S.; Valiev, M.; Dupuis, M.; Xantheas, S. S.; Sugiki, S.; Sekino, H. Mol. Phys. 2005, 103, 2255.

(50) Shiga, M.; Tachikawa, M. Mol. Simul. 2007, 33, 171.

(51) (a) Morita, A.; Kato, S. J. Chem. Phys. 1998, 109, 5511. (b) Li, S.; Schmidt, J. R.; Skinner, J. L. J. Chem. Phys. 2006, 125, 244507. 33.

(52) Humphrey, W.; Dalke, A.; Schulten, K. J. Mol. Graph. 1996, 14, 